\title{
ABO incompatibile graft management in pediatric transplantation
}

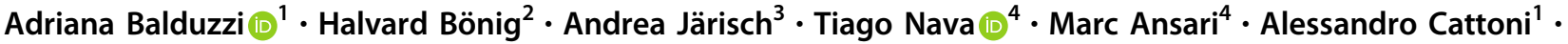 \\ Giulia Prunotto ${ }^{1}$ - Giovanna Lucchini ${ }^{5}$ - Gergely Krivan ${ }^{6} \cdot$ Toni Matic $^{7} \cdot$ Krzyzstof Kalwak $^{8}{ }^{8}$. Akif Yesilipek ${ }^{9}$. \\ Marianne Ifversen ${ }^{10} \cdot$ Peter Svec $^{11} \cdot$ Jochen Büchner $^{12} \cdot$ Kim Vettenranta $^{13} \cdot$ Roland Meisel $^{14}{ }^{14}$. Anita Lawitschka ${ }^{15}$. \\ Christina Peters $\mathbb{D}^{15} \cdot$ Brenda Gibson $^{16}$. Arnaud Dalissier ${ }^{17} \cdot$ Selim Corbacioglu ${ }^{18}$. André Willasch $\left(\mathbb{D}^{3}\right.$. \\ Jean-Hugues Dalle ${ }^{19} \cdot$ Peter Bader $^{3} \cdot$ on behalf of the EBMT Pediatric Diseases Working Party
}

Received: 20 June 2019 / Revised: 28 March 2020 / Accepted: 12 June 2020

(c) The Author(s), under exclusive licence to Springer Nature Limited 2020

\begin{abstract}
Up to $40 \%$ of donor-recipient pairs in SCT have some degree of ABO incompatibility, which may cause severe complications. The aim of this study was to describe available options and survey current practices by means of a questionnaire circulated within the EBMT Pediatric Diseases Working Party investigators. Major ABO incompatibility (donor's RBCs have antigens missing on the recipient's cell surface, towards which the recipient has circulating isohemagglutinins) requires most frequently an intervention in case of bone marrow grafts, as immediate or delayed hemolysis, delayed erythropoiesis and pure red cell aplasia may occur. RBC depletion from the graft (82\%), recipient plasma-exchange (14\%) were the most common practices, according to the survey. Graft manipulation is rarely needed in mobilized peripheral blood grafts. In case of minor incompatible grafts (donor has isohemagglutinins directed against recipient RBC antigens), isohemagglutinin depletion from the graft by plasma reduction/centrifugation may be considered, but acute tolerability of minor incompatible grafts is rarely an issue. According to the survey, minor ABO incompatibility was either managed by means of plasma removal from the graft, especially when isohemagglutinin titer was above a certain threshold, or led to no intervention at all (41\%). Advantages and disadvantages of each method are discussed.
\end{abstract}

\section{Introduction}

Blood group incompatibility between donors and recipients can be managed by means of graft/recipient manipulation. The potential impact of $\mathrm{ABO}$ incompatibility on transplantation outcomes ranges from no impact to severe complications.

A survey was conducted to investigate the current practice in relation to $\mathrm{ABO}$-incompatible transplantation within the EBMT Pediatric Diseases Working Party.

Members of the EBMT Pediatric Diseases Working Party are listed before references.

Adriana Balduzzi

abalduzzi@fondazionembbm.it

Extended author information available on the last page of the article

\section{Background}

\section{ABO blood groups}

ABO antigens are immunodominant sugars, naturally expressed at very high density $\left(2 \times 10^{6} /\right.$ cell $)$ on the surface of red blood cells (RBCs), platelets, white blood cells, vascular and organ endothelium and, in individuals of the Secretor phenotype, freely circulating in plasma. The enzymes transferring the sugars to a polysaccharide precursor structure called " $\mathrm{H}$ " are co-dominantly inherited; they are referred to as "A-transferase" and "B-transferase". Irrespective of the $\mathrm{ABO}$ genotype, expression of $\mathrm{A}$ or $\mathrm{B}$ antigens is contingent on the ability to generate $\mathrm{H}$ antigen; the rare individuals lacking an $\mathrm{H}$ transferase exhibit the Bombay phenotype and generate anti-H isohemagglutinins. Blood group $\mathrm{O}$ is characterized by inheritance of two nonfunctional variants of the A-transferase gene, typically a frameshift mutation leading to a premature stop codon; $\mathrm{RBCs}$ from blood group $\mathrm{O}$ individuals express unmodified $\mathrm{H}$ antigen. 
Anti-A and anti-B antibodies, commonly referred to as isohemagglutinins, are predominantly immunoglobulin $\mathrm{M}$ (IgM) naturally appearing in plasma early in the first year of life due to the high prevalence of $\mathrm{A}$ - and B-like polysaccharides on food and environmental microbes and are directed against A or B antigens lacking on the subject's RBCs, if any. Therefore subjects of blood group A express A antigens on their cell surface and invariably carry anti-B antibodies in their plasma, those of blood group B express B antigens on their cell surface and invariably carry anti-A antibodies in their plasma, those of blood group AB express $\mathrm{A}$ and $\mathrm{B}$ antigens on their cell surface and carry no isohemagglutinins in their plasma, and those of blood group $\mathrm{O}$ express no $\mathrm{ABO}$ antigens on their cell surface and carry both anti-A and anti-B antibodies in their plasma.

Unlike IgG, IgM antibodies can directly fix complement and cause immediate intra-vascular hemolysis of RBCs carrying the cognate antigen.

\section{ABO incompatibility}

ABO genes (chromosome 9) are inherited independently of HLA (chromosome 6). In the allogeneic setting, due to the skewed distribution of $\mathrm{ABO}$ blood groups in most populations, up to $40 \%$ of the donor/recipient pairs have some degree of $\mathrm{ABO}$ incompatibility, which has been classified as major, minor, or bidirectional [1, 2].

Major ABO incompatibility occurs when the donor's RBCs express A and/or B antigens, at least one of which is absent on the recipient's cell surface and who as a result has circulating isohemagglutinins against the donor RBCs. After the infusion of unmanipulated major ABO incompatible marrow, RBCs in the graft enter the recipient bloodstream. Recipient antibodies bind donor RBC antigens and activate the complement cascade with massive hemolysis, proportional to the amount of RBCs infused and the titer of recipient antibodies. Because of their relative frequencies, this scenario most commonly occurs when a blood group A graft is infused into a blood group $\mathrm{O}$ recipient.

Minor incompatibility occurs when the donor has isohemagglutinins, besides B-lymphocytes generating these, directed against recipient $\mathrm{RBC}$ antigens. After the infusion of unmanipulated minor $\mathrm{ABO}$ incompatible bone marrow, donor isohemagglutinins may cause hemolysis of recipient RBCs, proportional to the isohemagglutinin titer. This scenario most commonly occurs when a blood group $\mathrm{O}$ graft is infused into a blood group A recipient.

Bidirectional ABO incompatibility involves both the major and minor incompatibilities, i.e., infusion of a blood group B graft into a blood group A recipient or vice-versa.

Because ABO incompatibility can cause severe complications which jeopardize SCT outcome, donor blood group may influence donor choice.

\section{Impact of $\mathrm{ABO}$ incompatibility}

The infusion of unmanipulated major $\mathrm{ABO}$ incompatible marrow may cause immediate hemolysis, delayed hemolysis, delayed erythropoiesis and pure red cell aplasia. Pure red cell aplasia is caused by recipient isohemagglutinins inhibiting the normal erythroid precursors maturation at the colony-forming unit stage, which is the earliest stage of $\mathrm{ABO}$ antigen presentation. Delayed erythropoiesis/erythroid engraftment presents in the post-transplant course, with a low reticulocyte count, increased transfusional requirement, and, less frequently, delayed granulocyte and platelet engraftment [1-5].

The infusion of unmanipulated minor ABO incompatible marrow may cause early hemolysis, which is very rare, or, rarely, delayed (7-14 days) massive hemolysis secondary to passenger lymphocyte (producing isohemagglutinins) syndrome. Passenger lymphocyte syndrome cannot be prevented by plasma removal from the graft, to remove isohemagglutins, as B-lymphocytes remain into the graft, but may be prevented by in vivo rituximab [1-4].

Despite the management of ABO incompatibility by means of graft/recipient manipulation, the potential impact of major $\mathrm{ABO}$ incompatibility on transplantation outcomes ranges from no impact to delayed engraftment, increased transfusion requirement, graft failure, increased graftversus-host incidence and severity, increased transplantrelated mortality, and decreased overall survival.

A survey was conducted to investigate current practice in relation to ABO-incompatible transplantation. This was done by means of a questionnaire circulated to the members of the EBMT PD WP, on behalf of the Supportive Care Task Force.

\section{Subjects and methods}

\section{Survey amongst the PD WP supportive care task force}

Investigators from 29 centers participated into the survey among the 52 who had been invited to join the Supportive Care Task Force.

ABO compatibility was included among the donor selection criteria by $89 \%$ of the participating centers. However, major ABO incompatibility was only ranked as the sixth criterion in the list, after HLA compatibility, CMV serological status, stem cell source, donor age and donor gender. Minor ABO incompatibility was ranked as less important, after parity for female donors and donor center location, as shown in Table 1. Major ABO incompatibility was never judged as a criterion to reject a donor.

Specific yields of the survey are included in each section. 
Table 1 Hierarchical criteria for donor selection.

\begin{tabular}{|c|c|c|c|c|c|c|c|c|c|c|c|c|c|c|c|c|c|c|c|c|}
\hline & \multicolumn{2}{|c|}{$1 \mathrm{st}$} & \multicolumn{2}{|c|}{ 2nd } & \multicolumn{2}{|c|}{ 3th } & \multicolumn{2}{|c|}{ 4th } & \multicolumn{2}{|c|}{5 th } & \multicolumn{2}{|c|}{ 6th } & \multicolumn{2}{|c|}{ 7th } & \multicolumn{2}{|c|}{ 8th } & \multicolumn{2}{|c|}{ 9th } & \multirow{2}{*}{$\begin{array}{l}\text { Total } \\
N\end{array}$} & \multirow[t]{2}{*}{ Score } \\
\hline & $N$ & $\%$ & $N$ & $\%$ & $N$ & $\%$ & $N$ & $\%$ & $N$ & $\%$ & $N$ & $\%$ & $N$ & $\%$ & $N$ & $\%$ & $N$ & $\%$ & & \\
\hline 9/10 vs $10 / 10$ HLA compatibility & 28 & 97 & 0 & 0 & 0 & 0 & 0 & 0 & 1 & 3 & 0 & 0 & 0 & 0 & 0 & 0 & 0 & 0 & 29 & 8.9 \\
\hline CMV serological status (non $\mathrm{R}+/ \mathrm{D}-$ ) & 0 & 0 & 8 & 28 & 12 & 41 & 5 & 17 & 1 & 3 & 3 & 10 & 0 & 0 & 0 & 0 & 0 & 0 & 29 & 6.7 \\
\hline Stem cell source & 0 & 0 & 14 & 42 & 6 & 21 & 1 & 3 & 0 & 0 & 2 & 7 & 3 & 10 & 3 & 10 & 0 & 0 & 29 & 6.3 \\
\hline Donor age & 0 & 0 & 5 & 18 & 4 & 14 & 4 & 14 & 7 & 25 & 5 & 18 & 2 & 7 & 1 & 3 & 0 & 0 & 28 & 5.5 \\
\hline Donor gender & 0 & 0 & 0 & 0 & 5 & 17 & 11 & 38 & 4 & 14 & 3 & 10 & 4 & 14 & 1 & 3 & 1 & 3 & 29 & 5.1 \\
\hline Major ABO incompatibility & 0 & 0 & 0 & 0 & 0 & 0 & 4 & 14 & 7 & 24 & 6 & 20 & 9 & 31 & 3 & 10 & 0 & 0 & 29 & 4.0 \\
\hline Parity in case of female donor & 0 & 0 & 0 & 0 & 1 & 3 & 3 & 10 & 5 & 17 & 7 & 24 & 6 & 21 & 3 & 10 & 4 & 14 & 29 & 3.7 \\
\hline Donor center geografical location & 1 & 3 & 1 & 3 & 1 & 3 & 1 & 3 & 2 & 7 & 1 & 3 & 1 & 3 & 7 & 24 & 14 & 48 & 29 & 2.6 \\
\hline Minor ABO incompatibility & 0 & 0 & 1 & 3 & 0 & 0 & 0 & 0 & 2 & 7 & 2 & 7 & 3 & 10 & 11 & 38 & 10 & 34 & 29 & 2.3 \\
\hline
\end{tabular}

Donor selection criteria have been hierarchically ordered by the 29 investigators from the pediatric transplant centers participating to the EBMT PD WP Supportive Care Task Force. Possible criteria for donor selection are listed in the first column, by hierarchical order, resulting after the survey. In each box of the table the number (and percentage in italics) of investigators who ranked the specific criterion in that position is shown. A score is shown in the last column, resulting from the weighted mean of the overall ranking.

\section{Results}

\section{How to deal with ABO incompatibility}

When the donor and the recipient are of different $\mathrm{ABO}$ blood groups, $\mathrm{ABO}$ incompatibility has to be managed to maximize the chances of safe graft infusion and engraftment.

\section{Major incompatibility}

Different approaches apply to circumvent the issue of circulating isohemoagglutinin titers directed against donor $\mathrm{RBC}$ contained in the graft in case of major $\mathrm{ABO}$ incompatibility. Each strategy will aim at removing/reducing either donor $\mathrm{RBC}$ from the graft or circulating isohemagglutinin from the donor.

$R B C$ depletion of the graft is the most frequently used approach. Most common methods of RBC removal are sedimentation under gravity, after the addition of hydroxyethyl starch (HES), separation on a Ficoll gradient and processing by a blood cell separator. The blood cell separator within an automated system is progressively becoming more and more common in the most recent practice, allowing efficient RBC removal and limited cell loss $[4,6,7]$.

Cell loss is selective so that non-mononuclear cells (MNC) are mainly lost and most CD34+ cells are recovered. Nevertheless, MNC cell loss may be significant, especially when there is an unfavorable donor/recipient weight disparity. This is rarely an issue in pediatrics, except in the case of a younger sibling donating to a heavier older sibling. Fortunately, ABO incompatibility is less common in the related setting.
Despite RBC depletion, the residual RBC load in the graft may still be too high for low weight recipients. This can be addressed by diluting the graft in recipientcompatible packed $\mathrm{RBC}$, so that the residual RBCs are predominantly compatible [8].

In terms of residual incompatible RBC in the graft, no safety threshold level could be stated for avoidance of hemolysis on the basis of available data. As a practical rule, a residual volume of incompatible $\mathrm{RBCs}<50 \mathrm{~mL}$ in nearly adult weights or $<0.5 \mathrm{~mL} / \mathrm{Kg}$ is generally considered acceptable. The strategies mentioned above, sedimentation in HES, separation on a Ficoll gradient and processing by blood cell separators, allow a reduction in residual RBCs to well below this limit.

Depleting RBCs from the graft is essentially always successful, allows to avoid acute adverse reactions and is stem cell sparing. Moreover, RBC depletion allows to reduce the amount of heparin in the graft, which may render the blood temporarily uncoagulable in a low weight child, as well as its volume, which is of benefit, particularly in younger children.

Nevertheless, by means of RBC depletion, recipient isohemagglutinins remain in the patient and will likely attack the new graft-derived RBCs and lyse an amount of RBCs equivalent to the RBC units which would have been needed to reduce the isohemagglutinin titer with a pretransplant plasma exchange. Therefore persistent anemia, resembling a delayed erythroid engraftment can be expected after a major $\mathrm{ABO}$ incompatible graft manipulated with RBC depletion, sometimes for months, up to when all isohemagglutinins will have adsorbed to the newborn RBCs.

Plasma exchange of the recipient prior to the graft infusion performs an "in vivo isohemagglutinin depletion". 
It has the advantages of avoiding any cell loss and graft manipulation and has been described by Sheppard et al in 34 patients [9]. The disadvantages include the discomfort of patients, who may be critically ill, the logistics may be an issue, the possible need for multiple procedures and the requirement for considerable expertise, particularly in low weight children. Finally, the method is relatively expensive and time-consuming and may not result in a sufficient isohemagglutinin titer reduction, recognizing that an acceptable titer remains a matter of debate $(<1: 16 ?<1: 32$ ?). The conceptual advantage is that the removal of isohemagglutinins prevents the impediment of RBC engraftment, unlike graft depletion. Such an approach may reduce the incidence of pure red cell aplasia (from 16 to $6 \%, p$ value 0.009) [10].

Pre-emptive repeated recipient transfusion of donor-type $R B C$ in the recipient aims at adsorbing recipient isohemagglutinins and is referred to as whole blood immunoadsorption performed during the conditioning regimen [11]. This approach can be considered a sort of in vivo isohemagglutinin depletion and has the advantage of avoiding graft manipulation and cell loss. It is expected that recipient-type RBCs are quantitatively lysed with the release of free hemoglobin (highly toxic, quencher of NO) and membrane fragments (inducers of disseminated intravascular coagulation) into the blood stream. Therefore it carries the disadvantage of potentially significant RBC transfusion-related reactions (fever, hemoglobinuria, hemolysis), with exceptional cases of renal failure being reported (hyperhydration and pre-medication recommended).

A retrospective study on 35 adult patients reported that donor type RBC transfusion before peripheral blood stem cell transplantation in the major ABO incompatibility setting was relatively safe. No complications had been described in $66 \%$ of the patients, but $23 \%$ of the patients experienced mild effects and $11 \%$ of them experienced severe adverse events, described overall as chest pain, headache, high changes in heart-rate, blood pressure, $\mathrm{O} 2$ saturation, which led to the procedure discontinuation [11]. In the same study, a decrease of the isohemagglutinin titer by immunoadsorption and the reduction in RBC transfusion requirements in the first month after transplant ( $p$ 0.003), with an absence of pure red cell aplasia was reported [10], although the expected frequency of pure red cell aplasia is low. This experience has been reproduced in a single pediatric center (Järisch's personal communication). A previous experience [12] reported no complications in 12 patients, except in 1 patient who had a pre-existing renal impairment.

The process is expected to be roughly neutral with respect to allogeneic blood use, as the amount of RBCs infused pre-transplant to deplete isohemagglutinins may not be needed as transfusional support in the post-transplant period, as it will lower the titer of circulating isohemagglutinins which can attack newborn RBCs, which may ultimately engraft in the post-transplant course.

The acute safety and tolerability of donor-type RBC infusion should be demonstrated in a larger cohort; furthermore, pediatric patients may tolerate the procedure differently. A potential impairment of renal function may not have been specifically investigated and cannot be ruled out.

Pre-emptive recipient transfusion with donor type Secretor plasma is referred to as plasma immunoadsorption and involves the infusion of donor-type plasma, containing circulating RBC antigens [13], which recipient isohemagglutinins adsorb to. It has the advantage of avoiding graft manipulation and cell loss, without causing RBC hemolysis. The disadvantage is the lack of availability of Secretor plasma, because the Secretor phenotype is not routinely ascertained in blood donors. The benefit of this approach is not well established.

According to the survey, in case of bone marrow grafts, major $\mathrm{ABO}$ incompatibility was mainly managed by means of $82 \%$ of the investigators carried out RBC depletion of the graft, $14 \%$ a combination of RBC depletion and recipient plasma exchange, and a single-center favored pre-transplant donor RBC transfusions.

\section{Minor incompatibility}

Isohemagglutinin depletion of the graft, by a variety of means, may be considered. The simplest method is plasma reduction of the graft by centrifugation and exchange of donor plasma for recipient- and donor-compatible plasma or serum albumin. Most centers perform this only when the isohemagglutinin titer is above a threshold which varies between $1: 32$ to $1: 128$.

According to the survey, minor $\mathrm{ABO}$ incompatibility was either managed by plasma removal from the graft (52\%) or no intervention $(41 \%)$.

In a previous report within adult transplant centers, plasma depletion was performed in the vast majority of the centers, regardless of the titers $(81 \%)$, or only in case of a high titer (19\%), even though the threshold deeply varied, ranging from $1: 16$ to $1: 256$.

\section{ABO incompatibility management according to stem cell source}

Major incompatibility. RBCs constitute $25-35 \%$ of the bone marrow volume. When the infused marrow volume ranges between 8 and $20 \mathrm{~mL} / \mathrm{Kg}$, approximately $2-7$ $\mathrm{RBCmL} / \mathrm{Kg}$ are infused. 
By contrast, the RBC content is very low in technically well-performed mobilized peripheral blood apheresis grafts. Therefore peripheral blood apheresis products rarely require graft manipulation before infusion.

The issue of $\mathrm{ABO}$ major incompatibility has to be considered in poor quality apheresis from the technical point of view. An apheresis with a high hematocrit may cause a very severe hemolytic transfusion reaction, as previously reported [8] and would require similar approaches as in the bone marrow management.

Once again, even if PBSC very rarely require ex vivo RBC depletion, as no acute hemolysis is expected, given the low amount of donor RBCs contained in the graft, circulating recipient isohemagglutinins will act as described above in the first weeks after transplant, and potentially delay engraftment, particularly of the erythroid compartment.

According to the survey, when $\mathrm{PB}$ was the stem cell source, $62 \%$ of the centers undertook no interventions, $28 \%$ RBC removal of the graft and $10 \%$ other approaches or a combination of the ones listed above.

Cord bloods (CB) are collected and cryopreserved in small volumes, so that $\mathrm{ABO}$ incompatibility is usually irrelevant; it might be considered in very low-weight recipients, but not in neonatal age, when acquired immunity is starting and no anti-donor antibody exists. Expression of A/ $\mathrm{B}$ antigens is quite low on the neonatal RBC surface, so they are much less sensitive to recipient isohemagglutinins. Moreover, most CB are RBC-depleted, in order to reduce volume, optimize freezing conditions for mononuclear cells, avoid hemolysis and free hemoglobin in the final product and facilitate smooth thawing. Therefore RBC depletion should be one of the selection criteria for $\mathrm{CB}$ units, regardless of $\mathrm{ABO}$ incompatibility, which is not usually an issue in the $\mathrm{CB}$ setting. The passenger lymphocyte syndrome has never been described after CB transplantation, as neonatal $\mathrm{B}$-cells at birth are all naïve and have not been sensitized by $\mathrm{A} / \mathrm{B}$ antigens.

According to the survey, when $\mathrm{CB}$ was the stem cell source, $41 \%$ of the centers selected only RBC depleted units, whilst $34 \%$ accepted RBC-replete units but RBC depleted before infusion.

\section{Discussion}

ABO incompatible donor/recipient transplantation poses a unique challenge to the clinical team, the processing lab and the blood bank. It is essential that there is a good communication between these services actively interacting with one another to ensure patient safety.

This review attempted to focus on graft/recipient management in case of $\mathrm{ABO}$ incompatibility by means of a survey, after which, we could conclude that procedures to manage $\mathrm{ABO}$ incompatibility are relatively homogeneous, at least more than in the adult setting.

Different methodologies for managing major $\mathrm{ABO}$ incompatibility are practiced. Either RBC removal from the graft or, more rarely, isohemagglutinin removal from the recipient plasma, or both, are most commonly used to prevent or limit acute hemolysis of donor RBCs in the case of major $\mathrm{ABO}$ incompatibility, with donor-type $\mathrm{RBC}$ infusion having been reported by a single center. The fact that ex vivo RBC depletion was a clear favorite is probably due to the typically favorable donor-recipient weight ratio in pediatrics. Nevertheless, unadsorbed circulating isohemagglutinins may be responsible for later issues, namely late RBC/overall engraftment. Responses from adult transplant centers, as previously published, were very mixed, as the risk of stem cell loss is more often a crucial issue.

As acute tolerability of $\mathrm{ABO}$ minor incompatible grafts is normally good and the occurrence of passenger lymphocyte syndrome is rare and unpredictable, approaches to manage minor incompatibility both in the literature and according to the survey were quite variable. Meanwhile, donor plasma removal may be performed, by centrifugation, to prevent acute hemolysis of recipient RBCs, although it is hardly an issue in minor incompatibility, due to the low quantity of plasma and, hence, isohemagglutinins, in the graft, except for lowest weight children receiving large volumes.

Both donor RBC and plasma removal are usually adopted in bidirectional ABO incompatibility.

Each method has its advantages and disadvantages and there is no clear evidence to support a consensus and no comparative studies have been performed.

Besides the Authors, the following Investigators (in alphabetical order) contributed to the EBMT PD WP survey mentioned in this manuscript: Bella Bielorai ${ }^{20,21}$, Simone Cesaro ${ }^{22}$, Tamara Diesch ${ }^{23}$, Koji Kato $^{24}$, Sergio Gomez ${ }^{25}$, Karin Mellgren ${ }^{26}$, Julia Palma ${ }^{27}$, Claudia Rossig $^{28}$, Martin Sauer ${ }^{29}$, Petr Sedlacek ${ }^{30}$, Peter Shaw ${ }^{31}$, Kirk Schultz $^{32}$, Jerry Stein ${ }^{33}$, Güngör Tayfun ${ }^{34}$, Dominik Turkiewicz ${ }^{35}$, Jacekz Wachowiak ${ }^{36}$

${ }^{20}$ Department of Pediatric Hematology-Oncology, The Edmond and Lily Safra Children's Hospital, Sheba Medical Center, Ramat-Gan, Israel. ${ }^{21}$ Sackler School of Medicine, Tel-Aviv University, Tel-Aviv, Israel. ${ }^{22}$ Pediatric Hematology Oncology, Azienda Ospedaliera Universitaria Integrata, Verona, Italy. ${ }^{23}$ University Children's Hospital Basel, Division of Pediatric Oncology/Hematology, Basel, Switzerland. ${ }^{24}$ Haematology, Oncology \& Cardiovascular Medicine, Kyushu University Hospital, Fukuoka, Japan. ${ }^{25}$ Stem Cell Transplantation Unit, Hospital de Niños Sor María Ludovica, La Plata, Argentina. ${ }^{26}$ Institution for Clinical Sciences, Department of Paediatrics, Queen Silvia Children's Hospital, Gothenburg, Sweden. ${ }^{27}$ Stem Cell Transplant Unit, Hospital Luis Calvo Mackenna, Santiago, Chile. ${ }^{28}$ Department of Pediatric Hematology and Oncology, University Children's Hospital Muenster, Muenster, Germany. ${ }^{29}$ Department of Pediatric Hematology and Oncology, University Hospital, Hannover Medical School, Hannover, Germany. ${ }^{30}$ Department of Pediatric Hematology and Oncology, University Hospital Motol, Prague, Czech Republic. ${ }^{31}$ The Children's Hospital at Westmead, Oncology Unit, Westmead, Sydney, NSW, Australia. ${ }^{32}$ Department of Pediatrics, 
British Columbia Children's Hospital, British Columbia, Canada. ${ }^{33}$ Pediatric Hematology Oncology, Schneider Children's Medical Center of Israel, Sackler Faculty of Medicine, Tel Aviv University, Tel Aviv, Israel. ${ }^{34}$ Division of Stem Cell Transplantation, Department of Pediatrics, University Children's Hospital, Zürich, Switzerland. ${ }^{35}$ Children's Hospital, Skåne University Hospital, Lund, Sweden. ${ }^{36}$ Department of Pediatric Oncology, Hematology and Transplantology, University of Medical Sciences, Poznan, Poland.

Author contributions $\mathrm{AB}$ and $\mathrm{HB}$ wrote the manuscript, $\mathrm{AJ}, \mathrm{JHD}, \mathrm{RM}$ gave major contributions, PB initiated the Supportive Task Force within the EBMT Pediatric Diseases Working Party and AW coordinated it; all the authors contributed to the survey reported in the manuscript; all authors reviewed the manuscript and approved its final version.

\section{Compliance with ethical standards}

Conflict of interest The authors declare that they have no conflict of interest.

Publisher's note Springer Nature remains neutral with regard to jurisdictional claims in published maps and institutional affiliations.

\section{References}

1. Booth GS, Gehrie EA, Bolan CD, Savani BN. Clinical guide to ABO-incompatible allogeneic stem cell transplantation. Biol Blood Marrow Transpl. 2013;19:1152-8. https://doi.org/10.1016/ j.bbmt.2013.03.018.

2. Staley EM, Schwartz J, Pham HP. An update on ABO incompatible hematopoietic progenitor cell transplantation. Transfus Apher Sci. 2016;54:337-44. https://doi.org/10.1016/j.transci.2016.05.010.

3. Lapierre V, Kuentz M, Tiberghien P. Allogeneic peripheral blood hematopoietic stem cell transplantation: guidelines for red blood cell immuno-hematological assessment and transfusion practice. Société Française de Greffe de Moelle. Bone Marrow Transpl. 2000;25:507-12.

4. Raimondi R, Soli M, Lamparelli T, Bacigalupo A, Arcese W, Belloni M. Gruppo Italiano Trapianto di Midollo Osseo et al. ABO-incompatible bone marrow transplantation: a GITMO survey of current practice in Italy and comparison with the literature. Bone Marrow Transpl. 2004;34:321-9.
5. Rowley SD, Donato ML, Bhattacharyya P. Red blood cellincompatible allogeneic hematopoietic progenitor cell transplantation. Bone Marrow Transplant. 2011;46:1167-85. https://doi. org/10.1038/bmt.2011.135

6. Sorg N, Poppe C, Bunos M, Wingenfeld E, Hümmer C, Krämer $\mathrm{A}$, et al. Red blood cell depletion from bone marrow and peripheral blood buffy coat: a comparison of two new and three established technologies. Transfusion 2015;55:1275-82. https:// doi.org/10.1111/trf.13001.

7. Kim-Wanner SZ, Bug G, Steinmann J, Ajib S, Sorg N, Poppe C, et al. Erythrocyte depletion from bone marrow: performance evaluation after 50 clinical-scale depletions with Spectra Optia. BMC, J Transl Med. 2017;15:1 https://doi.org/10.1186/s12967017-1277-6

8. Bonig $\mathrm{H}$, Bug G. RBC depletion from a PBSC graft. Bone Marrow Transplant 2017;52:1078-9. https://doi.org/10.1038/bmt. 2017.72.

9. Sheppard D, Tay J, Bryant A, McDiarmid S, Huebsch L, Tokessy $\mathrm{M}$, et al. Major ABO-incompatible BMT: isohemagglutinin reduction with plasma exchange is safe and avoids graft manipulation. Bone Marrow Transpl. 2013;48:953-7. https://doi.org/10. 1038/bmt.2012.264.

10. Stussi G, Halter J, Bucheli E, Valli PV, Seebach L, Gmür J, et al. Prevention of pure red cell aplasia after major or bidirectional ABO blood group incompatible hematopoietic stem cell transplantation by pretransplant reduction of host anti-donor isoagglutinins. Haematologica. 2009;94:239-48. https://doi.org/10. 3324/haematol.13356.

11. Scholl S, Klink A, Mügge LO, Schilling K, Höffken K, Sayer HG. Safety and impact of donor-type red blood cell transfusion before allogeneic peripheral blood progenitor cell transplantation with major ABO mismatch. Transfusion. 2005;45:1676-83.

12. Nussbaumer W, Schwaighofer H, Gratwohl A, Kilga S, Schönitzer D, Nachbaur D, et al. Transfusion of donor-type red cells as a single preparative treatment for bone marrow transplants with major ABO incompatibility. Transfusion. 1995;35:592-5.

13. Curley C, Pillai E, Mudie K, Western R, Hutchins C, Durrant S, et al. Outcomes after major or bidirectional ABO-mismatched allogeneic hematopoietic progenitor cell transplantation after pretransplant isoagglutinin reduction with donor-type secretor plasma with or without plasma exchange. Transfusion. 2012;52:291-7. https://doi.org/10.1111/j.1537-2995. 2011.03295.x.

\section{Affiliations}

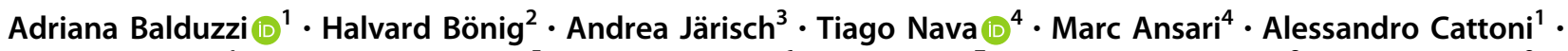

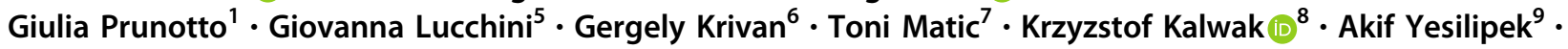 Marianne Ifversen ${ }^{10} \cdot$ Peter Svec $^{11} \cdot$ Jochen Büchner $^{12} \cdot$ Kim Vettenranta $^{13} \cdot$ Roland Meisel $^{14}{ }^{14}$ Anita Lawitschka ${ }^{15}$. Christina Peters $\mathbb{D}^{15}$. Brenda Gibson ${ }^{16}$. Arnaud Dalissier ${ }^{17}$. Selim Corbacioglu ${ }^{18}$. André Willasch $\mathbb{C}^{3}$. Jean-Hugues Dalle ${ }^{19} \cdot$ Peter Bader $^{3} \cdot$ on behalf of the EBMT Pediatric Diseases Working Party}

1 Clinica Pediatrica, Università degli Studi di Milano Bicocca, Fondazione Monza e Brianza per il Bambino e la sua Mamma, Ospedale San Gerardo, Monza, Italy

2 Blood Bank, Johann Wolfgang Goethe-Universität, Frankfurt am Main, Germany
3 Hospital for Children and Adolescents, Stammzelltransplantation und Immunologie, Klinik für Kinder- und Jugendmedizin, Johann Wolfgang Goethe-Universität, Frankfurt am Main, Germany

4 Division of Paediatric Onco-Hematology, University Hospital of Geneva, CANSEARCH Research Platform, Department of Child and Adolescent, University of Geneva, Geneva, Switzerland 
5 Stem Cell Transplantation Unit, Great Ormond Street Hospital, London, UK

6 Central Hospital of Southern Pest- National Institute of Hematology and Infectious Diseases, Budapest, Hungary

7 Department of Pediatrics, UHC Zagreb, Zagreb, Croatia

8 Department of Pediatric Hematology/Oncology and BMT, Wroclaw Medical University, Wroclaw, Poland

9 Antalya Medicalpark Hospital, Pediatric Stem Cell Transplantation Unit, Antalya, Turkey

10 Department of Pediatric and Adolescent Medicine, Rigshospitalet, Copenhagen University Hospital, Copenhagen, Denmark

11 Pediatric Hematology/Oncology, Division of Paediatric and Adolescent Medicine, Oslo University Hospital Rikshospitalet, Oslo, Norway
12 Bone Marrow Transplantation Unit, Bratislava, Slovakia

13 Hospital for Children and Adolescents, Helsinki University Central Hospital, Helsinki, Finland

14 Department of Pediatric Stem Cell Therapy, Pediatric Oncology, Hematology and Clinical Immunology, Heinrich-HeineUniversität Düsseldorf, Düsseldorf, Germany

15 St. Anna Children's Hospital, UKKJ, MUW, Vienna, Austria

16 Royal Hospital for Children, Glasgow, Scotland, UK

17 EBMT Paris Office, Hôpital Saint Antoine, Paris, France

18 Department of Pediatric Hematology, Oncology and Stem Cell Transplantation, University of Regensburg, Regensburg, Germany

19 Hemato-Immunology Department, Robert-Debre Hospital, APHP and Paris-Diderot University, Paris, France 
